PREPARED FOR THE U.S. DEPARTMENT OF ENERGY, UNDER CONTRACT DE-AC02-76CH03073

PPPL-3920

PPPL-3920

UC-70

Paul Trap Simulator Experiment to Model Intense

Beam Propagation in Alternating-gradient

Transport Systems

by

Erik P. Gilson, Ronald C. Davidson,

Philip C. Efthimion, and Richard Majeski

January 2004

NM|

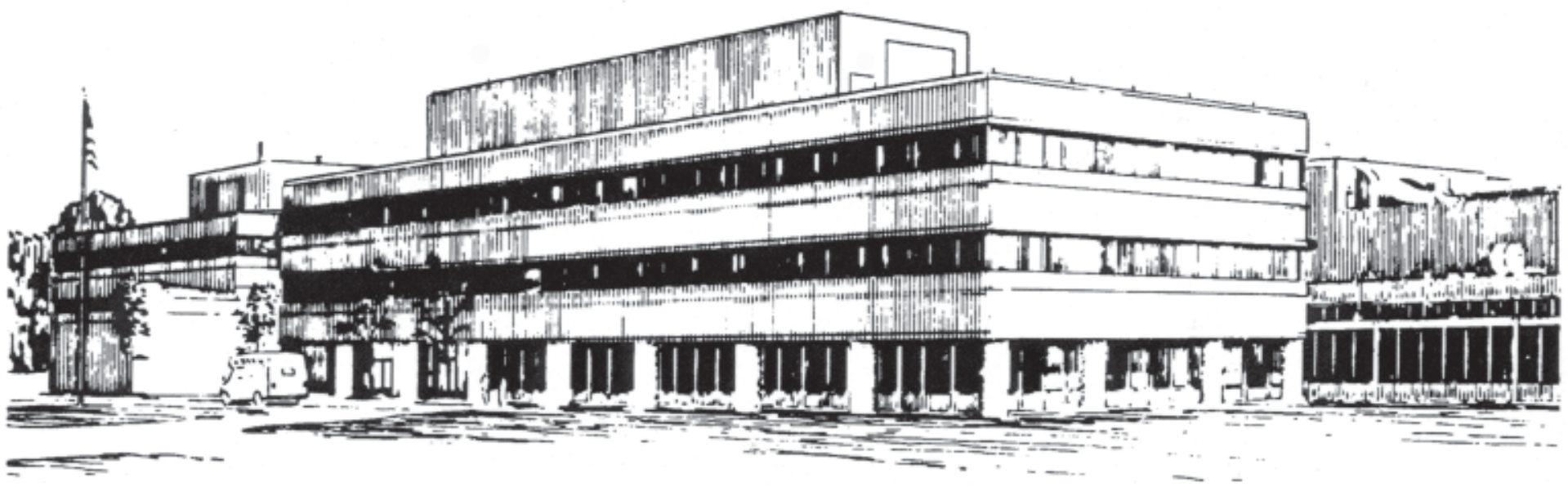

PRINCETON PLASMA PHYSICS LABORATORY PRINCETON UNIVERSITY, PRINCETON, NEW JERSEY 


\section{PPPL Reports Disclaimer}

This report was prepared as an account of work sponsored by an agency of the United States Government. Neither the United States Government nor any agency thereof, nor any of their employees, makes any warranty, express or implied, or assumes any legal liability or responsibility for the accuracy, completeness, or usefulness of any information, apparatus, product, or process disclosed, or represents that its use would not infringe privately owned rights. Reference herein to any specific commercial product, process, or service by trade name, trademark, manufacturer, or otherwise, does not necessarily constitute or imply its endorsement, recommendation, or favoring by the United States Government or any agency thereof. The views and opinions of authors expressed herein do not necessarily state or reflect those of the United States Government or any agency thereof.

\section{Availability}

This report is posted on the U.S. Department of Energy's Princeton Plasma Physics Laboratory Publications and Reports web site in Fiscal Year 2004. The home page for PPPL Reports and Publications is: http://www.pppl.gov/pub_report/

DOE and DOE Contractors can obtain copies of this report from:

U.S. Department of Energy

Office of Scientific and Technical Information

DOE Technical Information Services (DTIS)

P.O. Box 62

Oak Ridge, TN 37831

Telephone: (865) 576-8401

Fax: (865) 576-5728

Email: reports@adonis.osti.gov

This report is available to the general public from:

National Technical Information Service

U.S. Department of Commerce

5285 Port Royal Road

Springfield, VA 22161

Telephone: $1-800-553-6847$ or

(703) $605-6000$

Fax: (703) 321-8547

Internet: http://www.ntis.gov/ordering.htm 


\title{
Paul Trap Simulator Experiment to Model Intense Beam Propagation in Alternating-Gradient Transport Systems ${ }^{\dagger}$
}

\author{
Erik P. Gilson, ${ }^{*}$ Ronald C. Davidson, Philip C. Efthimion, and Richard Majeski \\ Plasma Physics Laboratory, Princeton University, Princeton, NJ 08543
}

(Dated: December 18, 2003)

\begin{abstract}
The results presented here demonstrate that the Paul Trap Simulator Experiment (PTSX) simulates the propagation of intense charged particle beams over distances of many kilometers through magnetic alternating-gradient (AG) transport systems by making use of the similarity between the transverse dynamics of particles in the two systems. Plasmas have been trapped that correspond to normalized intensity parameters $\hat{s}=\omega_{p}^{2}(0) / 2 \omega_{q}^{2} \leq 0.8$, where $\omega_{p}(r)$ is the plasmas frequency and $\omega_{q}$ is the average transverse focusing frequency in the smooth-focusing approximation. The measured root-mean-squared (RMS) radius of the beam is consistent with a model, equally applicable to both PTSX and AG systems, that balances the average inward confining force against the outward pressure-gradient and space-charge forces. The PTSX device confines one-component cesium ion plasmas for hundreds of milliseconds, which is equivalent to over $10 \mathrm{~km}$ of beam propagation.
\end{abstract}

PACS numbers: 52.59.Sa, 29.27.-a, 41.85.Ja, 52.27.Jt, Keywords: Ion Beam, Accelerator, Plasma, Paul Trap

Intense beam propagation [1-6] is an active area of research and is at the center of various scientific studies, including heavy ion fusion, spallation neutron sources, high energy physics, nonlinear dynamics, and nuclear waste transmutation. The results presented here demonstrate that the Paul Trap Simulator Experiment (PTSX) is capable of simulating, in a compact cylindrical Paul trap [7], beams with intensities up to $80 \%$ of the space-charge limit and that propagate for equivalent distances of over $10 \mathrm{~km}$. This allows the study of research topics such as: the conditions for quiescent beam propagation, collective mode excitation, generation and dynamics of halo particles, and distribution function effects. At the high beam intensities envisioned in present and next-generation facilities, a fundamental understanding of the influence of collective processes and self-field effects on beam transport and stability properties must be developed.

In intense beams, the space-charge effects are sufficiently strong that they affect the dynamics of the beam propagation. The strength of the space-charge force is characterized by the plasma frequency $\omega_{p}^{2}(r)=$ $n_{b} e_{b}^{2} / m_{b} \epsilon_{0}$, whereas the confining force is characterized by the average focusing frequency $\omega_{q}$ of the transverse oscillations of a particle in an AG system [1]. Here, $\epsilon_{0}$ is the permittivity of free space, $n_{b}(r)$ is the radial density profile, and $e_{b}$ and $m_{b}$ are the ion charge and mass, respectively. The normalized intensity parameter $\hat{s}=\omega_{p}^{2}(0) / 2 \omega_{q}^{2}$ describes whether the beam is emittance dominated $(\hat{s} \ll 1)$ or space-charge dominated $(\hat{s} \rightarrow 1)$. For example, Fermilab's Tevatron injector typically operates at $\hat{s} \sim 0.15$, and the Spallation Neutron Source is expected to operate at $\hat{s} \sim 0.2$. The PTSX device presently operates in the range $0 \leq \hat{s} \leq 0.8$.

Using a linear Paul trap to study beam dynamics was proposed by Davidson et al. [8] and by Okamoto and Tanaka [9]. The $q \boldsymbol{E}_{\perp}^{\text {ext }}$ forces that the PTSX electrodes exert on the trapped plasma particles are analogous to the $q \boldsymbol{v}_{\boldsymbol{z}} \times \boldsymbol{B}_{\perp}^{\text {ext }}$ forces that the AG system exert on the beam particles in the beam frame provided that long, coasting beams that are thin relative to the AG system magnet spacing are considered. Moreover, the self-field forces in both systems can be described by scalar potentials that obey Poisson's equation. In Ref. 8, it was shown that the self-consistent transverse Hamiltonians and the resulting Vlasov equations for the AG system and the PTSX system are equivalent, neglecting end effects. Thus, the good confinement properties of ions in PTSX and the arbitrary form of the voltage waveform applied to the confining electrodes make PTSX a compact, flexible laboratory facility in which to simulate intense beam propagation through AG systems.

In this paper, a description of the PTSX device is given, related theoretical analyses are summarized, and experimental results that examine the effects of varying the amount of injected plasma on the density profile and temperature of the trapped plasma are presented. These results are then compared with a global radial forcebalance model. Finally, the long-time confinement behavior of cesium ions in PTSX is described.

The PTSX device has been described elsewhere $[10,11]$ and only a brief description is presented here. The PTSX device (see Fig. 1) is a linear Paul trap and consists of three co-linear cylinders with radius $r_{w}=0.1 \mathrm{~m}$, each divided into four $90^{\circ}$ azimuthal sectors. The plasma is confined radially in the central $2 \mathrm{~m}$ long cylinder by oscillating voltages (typically $235 \mathrm{~V}$ at $75 \mathrm{kHz}$ ) applied as shown in Fig. 1. The outer two cylinders are each $0.4 \mathrm{~m}$ long and the voltage on these electrodes is held fixed at $150 \mathrm{~V}$ in order to confine the plasma axially. To inject or dump the ions, the voltage on one or the other set of outer electrodes is switched to the same oscillating voltage that is applied to the central cylinder. The time 
duration of injection $\left(t_{i}\right)$, trapping $\left(t_{t}\right)$, and dumping $\left(t_{d}\right)$ may be varied independently with typical values being $t_{i}=5 \mathrm{~ms}, t_{t} \leq 300 \mathrm{~ms}$, and $t_{d} \geq 10 \mathrm{~ms}$.
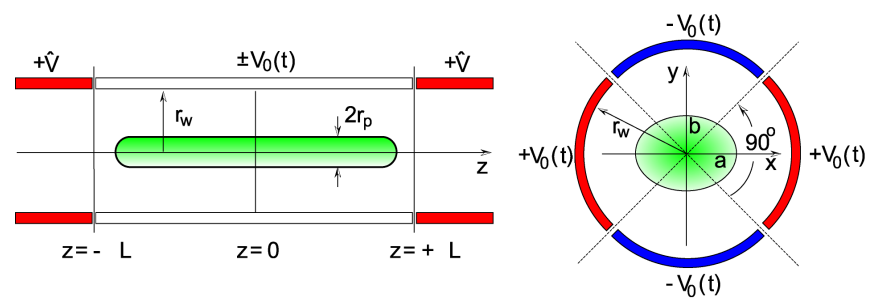

FIG. 1: The PTSX device consists of three cylindrical electrodes with radius $r_{w}=0.1 \mathrm{~m}$, each divided into four $90^{\circ}$ sectors. An oscillating voltage $\pm V_{0}(t)$ confines the plasma in the transverse plane to a radius $r_{p}$. Static voltages $+\hat{V}$ on the end electrodes confine the ions axially within a length $2 L=2 \mathrm{~m}$.

The cesium ion source consists of an aluminosilicate emitter surrounded by a Pierce electrode, followed by an acceleration grid and a deceleration grid to extract the desired ion current and adjust the final ion kinetic energy. On the dumping end of PTSX, there is a Faraday cup that is moveable in the transverse direction in order to measure the $z$-integrated radial charge profile. The operating pressure of PTSX is $5 \times 10^{-9}$ Torr.

The details of the analogy between AG systems and linear Paul traps such as PTSX are presented in Ref. 8. Here, we discuss the smooth-focusing transverse oscillation frequency $\omega_{q}$, the smooth-focusing vacuum phase advance $\sigma_{v}^{s f}$, and the force-balance equation that determines the RMS beam radius. The transverse motion of particles in either an AG system or in a Paul trap consists of rapid micromotion arising from the periodic focusing and defocusing forces, plus a guiding-center oscillation. In the smooth-focusing approximation, for sufficiently small vacuum phase advance $\sigma_{v}^{s f}$, the timescales of the two motions are well separated. The oscillation frequency of the average transverse motion $\omega_{q}$, neglecting space-charge effects, is then given by a simple analytic formula $[1,9]$. The change in phase of this transverse motion over one oscillation period of the focusing system is the smooth-focusing vacuum phase advance $\sigma_{v}^{s f}$.

In the PTSX system, transverse confinement can be described in terms of a ponderomotive force acting on the particles. For the circular PTSX electrodes, the applied electric potential near the axis at $r=0$ is [8]

$$
e_{b} \phi_{\mathrm{ap}}(x, y, t)=\frac{1}{2} \kappa_{q}(t)\left(x^{2}-y^{2}\right)
$$

where $\kappa_{q}(t)=8 e_{b} V_{0}(t) / m_{b} \pi r_{w}^{2}$. The voltage applied to the electrodes has the form $\pm V_{0}(t)= \pm V_{0} \max g(t)$, and $g(t)$ is a periodic function with unit amplitude and frequency $f$. For $r / r_{w} \ll 1$, the resulting ponderomotive force is proportional to the displacement from the axis, and the frequency of the transverse oscillations is given in the smooth-focusing approximation by $[1,10,11]$

$$
\omega_{q}=\frac{8 e_{b} V_{0 \max }}{m_{b} r_{w}^{2} \pi f} \xi
$$

where $m_{b}=133 \mathrm{amu}$ for $\mathrm{Cs}^{+}$ions in PTSX. The factor $\xi$ depends on the shape of the voltage waveform $g(t) ; \xi=1 / 2 \sqrt{2} \pi$ for a sinusoidal waveform, and $\xi=$ $4 \sqrt{3} /(\eta \sqrt{3-2 \eta})$ for a periodic step-function waveform with fill-factor $\eta$. Furthermore, the smooth-focusing vacuum phase advance $\sigma_{v}^{s f}$ is given by $\sigma_{v}^{s f}=\omega_{q} / f[1,10,11]$.

Under quasi-steady-state conditions, for a thermal equilibrium distribution of particles, the average density profile $n_{b}(r)$ is given by $[1,2]$

$$
n_{b}(r)=n_{b}(r=0) \exp \left[-\frac{m_{b} \omega_{q}^{2} r^{2}+2 e_{b} \phi^{s}(r)}{2 k T}\right] .
$$

Here, $k$ is Boltzmann's constant, $T=$ const. is the transverse temperature, and the space-charge potential $\phi^{s}(r)$ is determined self-consistently from Poisson's equation $r^{-1} \partial_{r}\left(r \partial_{r} \phi^{s}\right)=-n_{b}(r) e_{b} / \epsilon_{0}$. Manipulation of Eq. (3) gives the global radial force balance equation [1]

$$
m_{b} \omega_{q}^{2} R_{b}^{2}=2 k T+\frac{N_{b} e_{b}^{2}}{4 \pi \epsilon_{0}}
$$

where $N_{b}=\int_{0}^{r_{w}} n_{b}(r) 2 \pi r d r$ is the line density, and $R_{b}^{2}=\left(1 / N_{b}\right) \int_{0}^{r_{w}} n_{b}(r) 2 \pi r^{3} d r$ is the mean-squared radius of the plasma. As $R_{b}^{2}$ and $N_{b}$ are calculated from the measured data, $k T$ is the only parameter not known a priori.

For the data presented here, the voltage applied to the 0.6 inch diameter emitting surface of the cesium source was $9 \mathrm{~V}$, while the voltage on the acceleration grid was varied between $0 \mathrm{~V}$ and $8.7 \mathrm{~V}$. The deceleration grid was held fixed at $8 \mathrm{~V}$. The electrodes were driven with sinusoidal waveforms with a $235 \mathrm{~V}$ amplitude and a $75 \mathrm{kHz}$ frequency so that $\omega_{q}=6.51 \times 10^{4} \mathrm{~s}^{-1}$ and $\sigma_{v}^{s f}=49.7^{\circ}$.

The plasmas were injected for $5 \mathrm{~ms}$, trapped for $1 \mathrm{~ms}$, and then dumped. The radial charge profile, which is proportional to $n_{b}(r)$, is then measured by averaging the signal at each radial position over several hundred shots. The area of the Faraday cup aperture and an estimate of the plasma length $L_{p}[12]$ are then used to calculate $n_{b}(r)$. For example, the maximum on-axis charge shown in Fig. 2 corresponds to a density of $5 \times 10^{5} \mathrm{~cm}^{-3}$. Figure 2 shows the radial profiles measured for several acceleration grid voltages together with Gaussian fits to the radial profiles. From Eq. (3), plasmas that are in thermal equilibrium and are not exceptionally close to $\hat{s}=1$ are expected to have nearly Gaussian density profiles.

As the acceleration grid voltage is increased, more charge is injected into the trap. The profiles become taller and broader at first. Ultimately the profiles become shorter, while continuing to broaden. Note that a 
systematic error of $0.6 \mathrm{~cm}$ in the position of the Faraday cup has been removed from the data in Fig. 2, which is centered at $r=0$.

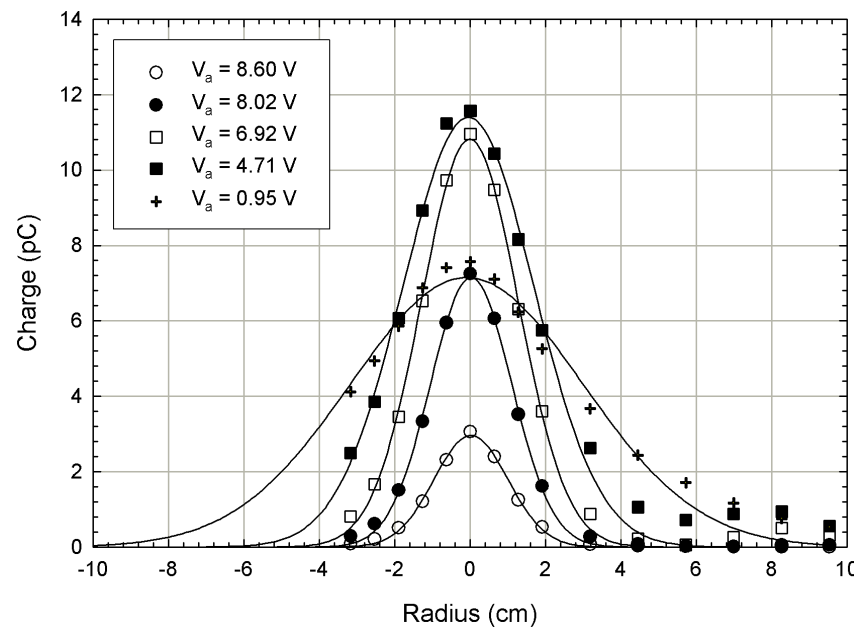

FIG. 2: Radial profiles of plasmas for various acceleration grid voltages show that by injecting more current into the trap, the peak height saturates and then decreases again.

To make comparisons with Eq. (4), $N_{b}$ is obtained from the experimental data either by: integrating the measured radial density profile $n_{b}(r)$ of a trapped plasma, or by knowing the energy and steady state current of a streaming beam reaching the Faraday cup enclosure and then invoking energy conservation plus the continuity equation [11]. These two methods produce values of $N_{b}$ that are proportional, but not identical; $N_{b}$ as computed from the streaming beam method is four times smaller. This is likely because the current collected on the Faraday cup enclosure may not be the entire current if the beam radius is larger than the enclosure and because the beam energy may not be immediately derivable from the ion source biases due to space-charge effects. Thus, the integrated radial profile technique is employed for calculating $N_{b}$.

The parameter $\hat{s}=\omega_{p}^{2}(0) / 2 \omega_{q}^{2}$ is now extracted from the measured density profile $n_{b}(r)$ by using the amplitude of the Gaussian fit $Q_{0}$. We note in Fig. 3 that as $N_{b}$ increases $\left(N_{b} \propto Q_{0} R_{b}^{2}\right.$ where $R_{b}$ is the width of the Gaussian fit), $\hat{s}$ initially increases, and reaches a plateau at $\hat{s}=0.8$. During the initial increase in $\hat{s}$, the radial density profiles become taller and somewhat broader. In the plateau region in Fig. 3, the radial profiles increase in width to accommodate the increasing amount of injected charge. For even larger values of $Q_{0} R_{b}^{2}$ than plotted in Fig. 3, the radial density profile continues to broaden and the on-axis density $n_{b}(0)$ and corresponding value of $\hat{s}$ decrease. This is likely due to the increasingly poor match between the plasma source radius and the trapped plasma radius at the higher values of injected charge.

Equation (4) informs us that the transverse temper-

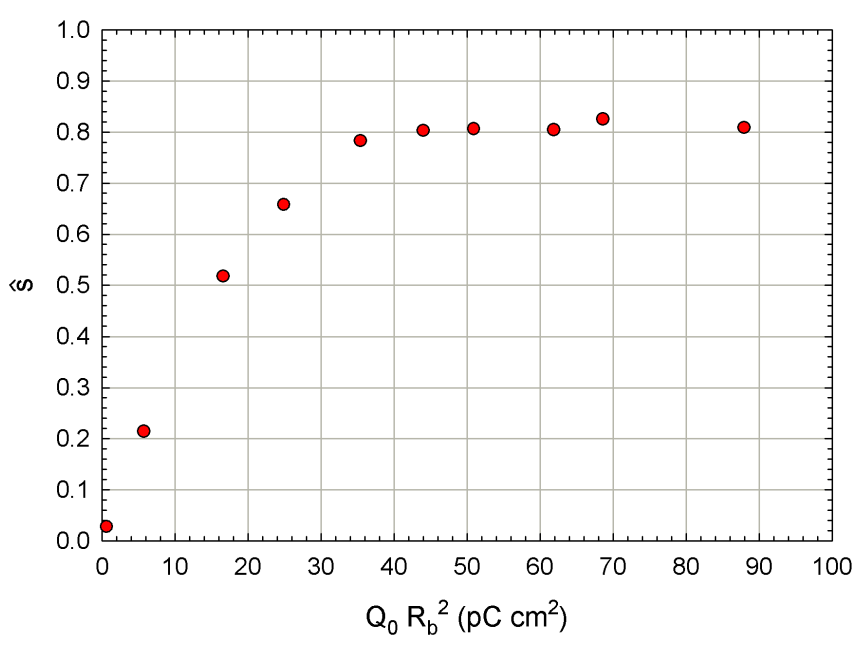

FIG. 3: The parameter $\hat{s}$ saturates at 0.8 before decreasing.

ature $k T$ (energy units) can be inferred by plotting $m_{b} \omega_{q}^{2} R_{b}^{2}$ versus $N_{b} e_{b}^{2} / 4 \pi \epsilon_{0}$. Figure 4 shows that for the region of increasing $\hat{s}, k T$ is approximately $0.5 \mathrm{eV}$. The data then depart from a straight line, consistent with the possible increase in transverse temperature once $\hat{s}$ becomes saturated, yet more charge is injected into the trap. The increasing mismatch between the source conditions and the trapped plasma likely serves as a source of free energy for transverse heating of the plasma (increase in emittance).

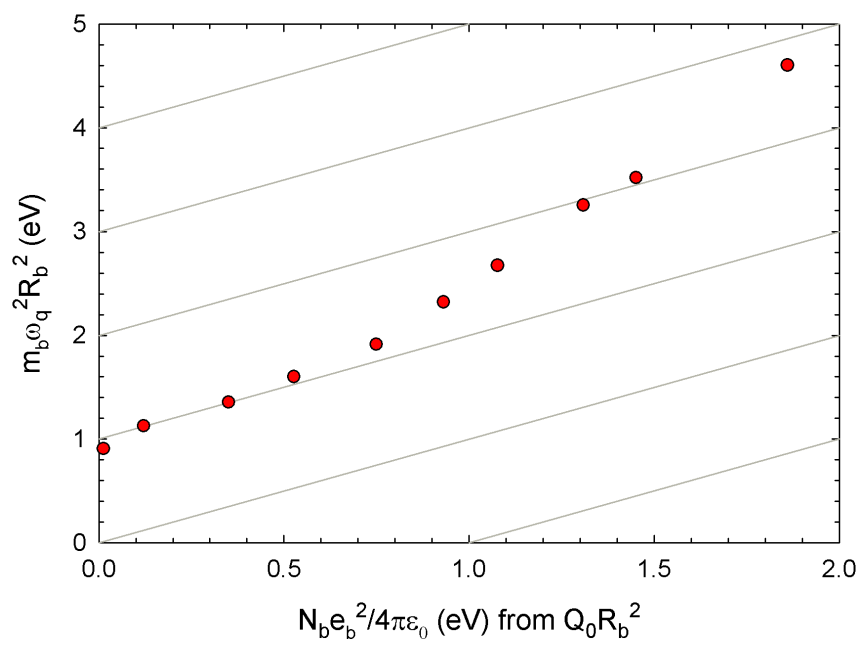

FIG. 4: The straight lines are plots of Eq. (4) for $2 k T=$ $0,1,2 \ldots \mathrm{eV}$ and are a guide to the reader. For small values of the line density $N_{b}$, the relationship is linear and implies a transverse temperature of $0.5 \mathrm{eV}$.

A temperature of $0.5 \mathrm{eV}$ is greater than expected, given that the ions are created with the same temperature as the aluminosilicate ion source $(k T \sim 0.1 \mathrm{eV})$. There are two likely causes for this heating. First, the deflection of the ion trajectories as they pass by the grid wires may contribute to an increase in the effective transverse tem- 
perature. Second, a fixed circular cross-section beam is not matched to an oscillating quadrupole configuration and this mismatch is a source of transverse heating.

The dependence of $R_{b}^{2}$ on $\omega_{q}^{2}$ in Eq. (4) can also be tested. Parameters are chosen so that $N_{b} e_{b}^{2} / 4 \pi \epsilon_{0} \ll k T$, and then $V_{0 \text { max }}$ and $f$ are adjusted to vary $\omega_{q}$ while $\sigma_{v}^{s f}$ is kept fixed. The results are shown in Fig. 5, where the solid curve is a plot of $R_{b}^{2}=2(0.5 \mathrm{eV}) / m_{b} \omega_{q}^{2}$. A temperature of $0.5 \mathrm{eV}$ is assumed because it is the inferred temperature of the data point in Fig. 5 for which $\omega_{q}^{2}=4.2 \times 10^{9} \mathrm{~s}^{-2}$. The agreement is reasonably good at smaller values of $\omega_{q}^{2}$, whereas the data lie well above the theoretical prediction at larger values of $\omega_{q}^{2}$. This is perhaps because the radius of the ion source cannot always be same as the radius of the trapped plasma, and this mismatch may also be a source of free energy to heat the plasma. It would then no longer be appropriate to assume $k T=0.5 \mathrm{eV}$ for all $\omega_{q}^{2}$ in making the comparison between theory and experiment.

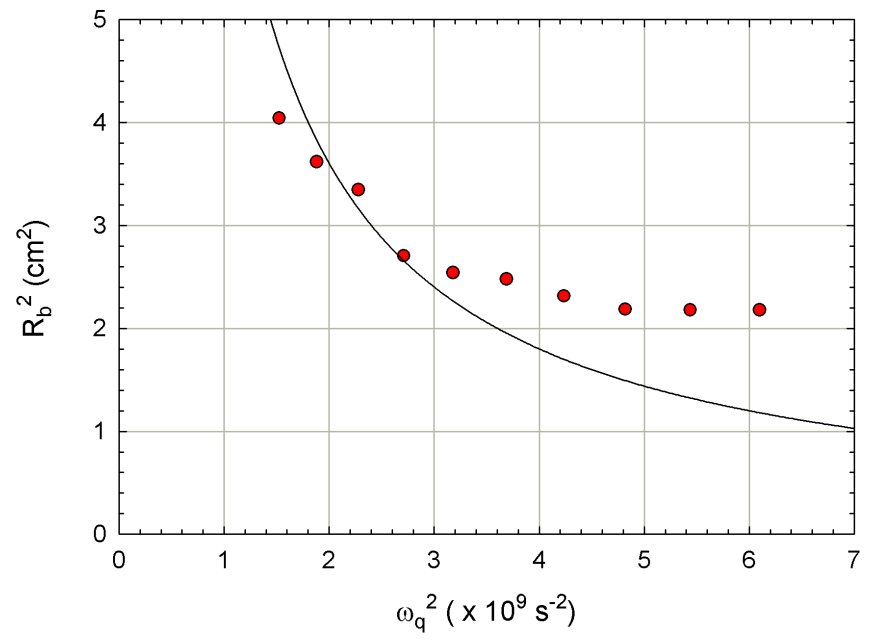

FIG. 5: The predicted hyperbolic relationship between $R_{b}^{2}$ and $\omega_{q}^{2}$ is shown by the solid curve assuming $k T=0.5 \mathrm{eV}$.

The PTSX device has the ability to confine ions for hundreds of milliseconds without distortion of the radial profile. Figure 6 demonstrates that a plasma with intensity parameter $\hat{s}=0.18$ is maintained for just over $300 \mathrm{~ms}$. It is important to realize that for a frequency $f=75 \mathrm{kHz}$ this corresponds to an accelerator system over $20 \mathrm{~km}$ long if the magnet lattice spacing is $1 \mathrm{~m}$. Furthermore, these plasmas are collisionless on timescales of tenths of seconds. For $n_{b}=5 \times 10^{5} \mathrm{~cm}^{-3}$ and $k T=0.5 \mathrm{eV}$, the ion-ion collision time is several seconds and for a background gas pressure of $5 \times 10^{-9}$ Torr, the ion-neutral collision time is tens of seconds.

In summary, the compact and flexible PTSX laboratory facility can simulate beam propagation for normalized intensities and equivalent propagation distances not readily accessible in many AG systems. Values of the normalized intensity parameter $\hat{s}$ up to 0.8 have been
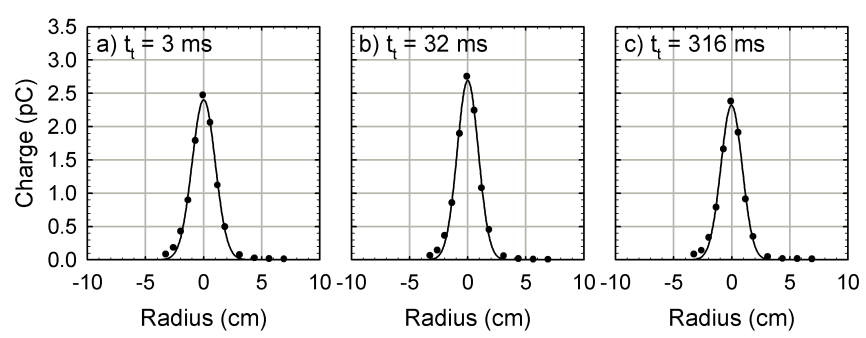

FIG. 6: The radial profile of a trapped plasma with intensity parameter $\hat{s}=0.18$ is only slightly degraded after $316 \mathrm{~ms}$.

achieved and the experimental data are in good agreement with a simple force balance model for a range of system parameters where the mismatch between the plasma source and the trapped plasma is not too large. Equivalent propagation distances over $10 \mathrm{~km}$ have been demonstrated. This validation of PTSX as a simulator of AG systems allows PTSX to be used to study beam propagation over large distances, collective mode excitation, halo particle effects, and distribution function effects such as multiple beamlets in a single transport system.

${ }^{\dagger}$ This research was supported by the U.S. Department of Energy.

* Electronic address: egilson@pppl.gov

[1] R. C. Davidson and H. Qin, Physics of Intense Charged Particle Beams in High Intensity Accelerators (World Scientific, Singapore, 2001).

[2] M. Reiser, Theory and Design of Charged Particle Beams (Wiley, New York, 1994).

[3] A. W. Chao, Physics of Collective Beam Instabilities in High Energy Accelerators (Wiley, New York, 1993).

[4] N. Kjærgaard and M. Drewsen, Phys. Plasmas 8, 1371 (2001).

[5] See, for example, Proceedings of the 2001 Particle Accelerator Conference (IEEE Catalog Number 01CH37268), pp. 1-4098.

[6] P. G. O'Shea et al., Nucl. Instrum. and Methods A 464, 646 (2001).

[7] W. Paul and H. Steinwedel, Z. Naturforsch. A 8, 448 (1953).

[8] R. C. Davidson, H. Qin, and G. Shvets, Phys. Plasmas 7, 1020 (2000).

[9] H. Okamoto and H. Tanaka, Nucl. Instrum. and Methods A 437, 178 (1999).

[10] E. P. Gilson, R. C. Davidson, P. C. Efthimion, R. Majeski, and H. Qin, Laser and Particle Beams 22, in press (2003).

[11] E. P. Gilson, R. C. Davidson, P. C. Efthimion, R. Majeski, and H. Qin, Proceedings of the 2003 Particle Accelerator Conference, in press (2003).

[12] E. P. Gilson, R. C. Davidson, P. C. Efthimion, R. Majeski, and E. A. Startsev, American Institute of Physics Conference Proceedings 692, 211 (2003). 


\section{External Distribution}

Plasma Research Laboratory, Australian National University, Australia

Professor I.R. Jones, Flinders University, Australia

Professor João Canalle, Instituto de Fisica DEQ/IF - UERJ, Brazil

Mr. Gerson O. Ludwig, Instituto Nacional de Pesquisas, Brazil

Dr. P.H. Sakanaka, Instituto Fisica, Brazil

The Librarian, Culham Laboratory, England

Mrs. S.A. Hutchinson, JET Library, England

Professor M.N. Bussac, Ecole Polytechnique, France

Librarian, Max-Planck-Institut für Plasmaphysik, Germany

Jolan Moldvai, Reports Library, Hungarian Academy of Sciences, Central Research Institute for Physics, Hungary

Dr. P. Kaw, Institute for Plasma Research, India

Ms. P.J. Pathak, Librarian, Institute for Plasma Research, India

Ms. Clelia De Palo, Associazione EURATOM-ENEA, Italy

Dr. G. Grosso, Instituto di Fisica del Plasma, Italy

Librarian, Naka Fusion Research Establishment, JAERI, Japan

Library, Laboratory for Complex Energy Processes, Institute for Advanced Study, Kyoto University, Japan

Research Information Center, National Institute for Fusion Science, Japan

Dr. O. Mitarai, Kyushu Tokai University, Japan

Dr. Jiangang Li, Institute of Plasma Physics, Chinese Academy of Sciences, People's Republic of China

Professor Yuping Huo, School of Physical Science and Technology, People's Republic of China

Library, Academia Sinica, Institute of Plasma Physics, People's Republic of China

Librarian, Institute of Physics, Chinese Academy of Sciences, People's Republic of China

Dr. S. Mirnov, TRINITI, Troitsk, Russian Federation, Russia

Dr. V.S. Strelkov, Kurchatov Institute, Russian Federation, Russia

Professor Peter Lukac, Katedra Fyziky Plazmy MFF UK, Mlynska dolina F-2, Komenskeho Univerzita, SK-842 15 Bratislava, Slovakia

Dr. G.S. Lee, Korea Basic Science Institute, South Korea

Institute for Plasma Research, University of Maryland, USA

Librarian, Fusion Energy Division, Oak Ridge National Laboratory, USA

Librarian, Institute of Fusion Studies, University of Texas, USA

Librarian, Magnetic Fusion Program, Lawrence Livermore National Laboratory, USA

Library, General Atomics, USA

Plasma Physics Group, Fusion Energy Research Program, University of California at San Diego, USA

Plasma Physics Library, Columbia University, USA

Alkesh Punjabi, Center for Fusion Research and Training, Hampton University, USA

Dr. W.M. Stacey, Fusion Research Center, Georgia Institute of Technology, USA

Dr. John Willis, U.S. Department of Energy, Office of Fusion Energy Sciences, USA

Mr. Paul H. Wright, Indianapolis, Indiana, USA 
The Princeton Plasma Physics Laboratory is operated by Princeton University under contract with the U.S. Department of Energy.

\author{
Information Services \\ Princeton Plasma Physics Laboratory \\ P.O. Box 451 \\ Princeton, NJ 08543
}

Phone: 609-243-2750

Fax: 609-243-2751

e-mail: pppl_info@pppl.gov

Internet Address: http://www.pppl.gov 